Research Paper

\title{
Dosimetric Effects of Head and Neck Immobilization Devices on Multi-field Intensity Modulated Radiation Therapy for Nasopharyngeal Carcinoma
}

Li Chen ${ }^{*}$, Ying-Lin Peng ${ }^{1 *}$, Shi-Yong Gu², Hui Shen ${ }^{3}$, Dan-Dan Zhang1, Wen-Zhao Sun ${ }^{1}$, Jian-Hua Wu ${ }^{1}$, Xiao-Wu Deng ${ }^{1 凶}$

1. Department of Radiation Oncology, Sun Yat-sen University Cancer Center, State key Laboratory of Oncology in South China, Collaborative Innovation Center for Cancer Medicine, Guangzhou 510060, China;

2. Department of Radiation Oncology, Wuhan General Hospital of Guangzhou Military, No. 627, Wuluo Road, Hongshan District, Wuhan 430064, China;

3. Department of Radiation Oncology, Yangjiang Hospital, No.42, Dongshan Road, Jiangcheng District, Yangjiang 529599, China.

*These authors contributed equally to the paper.

$\triangle$ Corresponding author: Xiaowu Deng, Department of Radiation Oncology, Sun Yat-sen University Cancer Center, State key Laboratory of Oncology in South China, Collaborative Innovation Center for Cancer Medicine, Guangzhou 510060, China. Telephone: +86-20-87343036 Fax: +86-20-87343394 E-mail: dengxw@mail.sysu.edu.cn

(c) Ivyspring International Publisher. This is an open access article distributed under the terms of the Creative Commons Attribution (CC BY-NC) license (https://creativecommons.org/licenses/by-nc/4.0/). See http://ivyspring.com/terms for full terms and conditions.

Received: 2018.01.11; Accepted: 2018.05.01; Published: 2018.06.14

\begin{abstract}
Background: In practice, the dose perturbation effect of head and neck immobilization devices is often overlooked in intensity-modulated radiation therapy (IMRT) for nasopharyngeal carcinoma (NPC). Purpose of this study is to verify and analyze the dosimetric effect of head and neck immobilization devices on NPC multi-field IMRT.

Methods: Ten patients with nasopharyngeal carcinoma were randomly selected. Two sets of body contours were established for each patient. One set of body contours did not contain the immobilization device, and the other contour set included the immobilization device. For each patient, dose calculations were conducted for the two sets of contours using the same 9-field IMRT plan, which were recorded as Plan- and Plan+. The dose difference caused by the head and neck immobilization devices was assessed by comparing the dose-volume histogram (DVH) parameter results and by plan subtraction. The gafchromic EBT3 film and anthropomorphic phantom were used to verify the calculated doses.

Results: The target coverage and average dose of Plan+ were lower than those of Plan- : the prescription dose coverage rates for PTVnx, PTVnd, PTV1 and PTV2 decreased by $2.4 \%, 9.9 \%, 1.5 \%$, and $3.6 \%$, respectively, and the mean doses were reduced by $0.9 \%, 1.9 \%, 1.1 \%$, and $1.5 \%$, respectively. Doses in the organs at risk showed no significant differences or slight reductions (the maximum reduction in mean dose was $1.7 \%$ ). From the EBT3 measurements, the skin dose on the posterior neck was increased by approximately $53 \%$.

Conclusion: The attenuation and bolus effects of the head and neck immobilization device reduce dose coverage rate and average dose of the planning target volumes in nasopharyngeal carcinoma and lead to an increase in the skin dose. During treatment planning and dose calculation, the immobilization device should be included within body contour to account for the dose attenuation and skin dose increment.
\end{abstract}

Key words: Head and neck immobilization device; Nasopharyngeal carcinoma; Intensity modulated radiation therapy; Dose attenuation; Skin dose

\section{Introduction}

Immobilization devices are widely used in radiation therapy to ensure accurate positioning and repositioning of patients during their fractionated radiotherapy [1-2]. Intensity modulated radiation therapy (IMRT) is the main radiotherapeutic technique used for nasopharyngeal carcinoma (NPC) 
[3-5]. When conducting intensity modulation planning and dose calculations, the commercial treatment planning system (TPS) calculate dose distributions only for the materials contained in the body structure [6,7]. Therefore, TPS usually requires the clarification of the body contour of the patient by manual/automatic delineation or by setting physical density threshold. In actual clinical practice, immobilization equipment is generally not outlined or calculated into the body contour. However, because the materials used in treatment couch top and immobilization devices are not equivalent to air, attenuation and scattering can occur when the radiation bean pass crosses these immobilization systems $[8,9]$. Several studies have demonstrated that treatment couch tops induce considerable beam attenuation, ranging from 4 to 9\% [10-12]. Immobilization devices used in radiotherapy may result in reduced tumor dose, increased skin dose and altered dose distribution [13-15]. Recently, American Association of Physicists in Medicine (AAPM) Task Group 176 recommended the dosimetric perturbations caused by the couch top and immobilization devices should be included in dose calculations whenever possible [16]. But no study has yet addressed the dosimetric effects of head and neck immobilization devices on the delivered doses to the target volume and to the organs at risk (OARs) for NPC IMRT. In this study, we investigated the effect of commercial immobilization device for NPC on dose distributions delivered with 9-filed IMRT technique. We compared the dose distribution calculated with the head and neck immobilization to that calculated without it. And the EBT3 films were calibrated and used to measure the skin dose of posterior neck for the situation with the immobilization devices enclosed. The dose-volumetric parameters and dose difference distribution map were calculated and compared to analyze the dosimetric effects of head and neck immobilization devices.

\section{Materials and Methods}

\section{Patient data and image acquisition}

Ten patients with nasopharyngeal carcinoma who received IMRT in our center were randomly selected and enrolled in this study. All patients were pathologically diagnosed and had no contraindications for radiotherapy. The patient sample included 5 males and 5 females, aged from 32 to 74 years, with a median age of 49.3 years. There were 5 cases of stage T3 and 5 cases of stage T4 carcinomas. All patients were immobilized using a head and neck fixation equipment customized for each patient body shape. This immobilization equipment (Klarity Inc, Guangzhou, China) included a carbon fiber floor, styrofoam bag, head/neck and shoulder thermoplastic film (Figure 1A). Its position fixation effect and repeatability were well verified in our previous study [3]. During simulation and treatment, all patients assumed the same supine position. A large aperture CT Simulation device (Brilliance, Philips Medical System, Amsterdam, Netherlands) was used to collect each patient CT images. The scan range was from the head to $2 \mathrm{~cm}$ below the sternum, and the slice thickness was $3 \mathrm{~mm}$. The obtained simulation CT images were sent to the treatment planning system (Monaco V5.1, Elekta AB, Stockholm, Sweden) via the radiotherapy network for treatment planning.

\section{Regions of interest}

The gross target volume of the nasopharynx (GTVnx) and that of the cervical lymph node (GTVnd), the clinical target volume of high-risk lymphatic drainage (CTV1) and the clinical target volume of lymphatic drainage for preventative radiation (CTV2) were delineated on CT images of all patients by experienced oncologists according to ICRU reports 50 and 62 [17,18]. The corresponding planning target volumes (PTVs) were generated by expanding $3 \mathrm{~mm}$ from CTVs symmetrically, and were recorded as PTVnx, PTVnd, PTV1 and PTV2 respectively. The delineated OAR included the brainstem, spinal cord, lens, optic nerve, optic chiasm, pituitary, parotid, inner ear, middle ear, temporal lobe, temporomandibular joint, mandible, tongue and throat. The spinal cord and brainstem areas were expanded by $3 \mathrm{~mm}$ to form the corresponding planning organ at risk volume (PRV). As the Monaco system calculates the dose distribution only for the area inside the body structure, we created two different body structure sets for each patient to investigate the dosimetric effects of the head and neck immobilization device. The first body structure set included only the patient body without the immobilization device. The other body structure set included the patient external body contours and the whole head and neck immobilization device.

\section{Treatment Planning and dose calculation}

The prescription doses to PTVnx, PTVnd, PTV1 and PTV2 were 70 Gy, 64-66 Gy, 60 Gy and 54 Gy, respectively, with a total of 30 fractions. The dynamic IMRT plans were generated without the head and neck immobilization devices included in the external body and recorded as Plan- in this study. The Planplans were created using 9 evenly distributed coplanar beams (every $40^{\circ}$, avoiding $180^{\circ}$ ) with $6 \mathrm{MV}$ X-rays. A Monte Carlo algorithm model was used to calculate the dose. The calculation model was commissioned and verified according to the report of AAPM Task Group 53. The maximum number of 
control points in each beam was limited to 18 . The computational grid size was $3 \times 3 \times 3 \mathrm{~mm}$. The statistical uncertainty of each control point was $3 \%$.

The electron density range of the whole head and neck immobilization device (0.1-1.28) was within the range of the TPS CT-ED calibration curve (0.1-1.69), so we thought the TPS had the ability to calculate the dose in the immobilization device. For each patient, the Plan- was then transplanted onto his/her own CT images with the external body contour containing the immobilization device. Under the same irradiation conditions, the dose distribution was recalculated and this new plan was recorded as Plan+.

\section{Dose difference analysis}

Dose-volume histogram (DVH) was used to evaluate the doses of the PTVs and the OARs for the same patient. The calculated parameters for the PTVs were the mean dose $\left(D_{\text {mean }}\right)$, the target coverage rate (CR) with the prescribed dose, the homogeneity index (HI) and the conformity index (CI). CR was calculated as follows:

$$
\mathrm{CR}=\mathrm{V}_{\mathrm{RX}} / \mathrm{V}_{\mathrm{T}} \times 100 \% \text { (1) }
$$

where $V_{T}$ is the volume of the target, and $V_{R X}$ is the target volume within the isodose curve (the reference isodose in this study was $100 \%$ of the prescribed dose).

$\mathrm{HI}$ and $\mathrm{CI}$ were respectively calculated as follows $[19,20]$ :

$$
\mathrm{HI}=\left(\mathrm{D}_{2 \%}-\mathrm{D}_{98 \%}\right) / \mathrm{D}_{50}(2)
$$

In formula (2), $\mathrm{D}_{2} \%, \mathrm{D}_{98 \%}$ and $\mathrm{D}_{50 \%}$ were the doses received by $2 \%, 98 \%$ and $50 \%$ of the volume of the Region of Interest (ROI), respectively. According to ICRU83, $\mathrm{D}_{2 \%}$ and $\mathrm{D}_{98 \%}$ represent the near-maximum dose and near-minimum dose, respectively [21]. A smaller HI value (the closer to 0) indicates better uniformity of the dose in the target area, whereas a higher $\mathrm{HI}$ indicates a less uniform target dose.

$$
\mathrm{CI}=\frac{\mathrm{V}_{\mathrm{RX}}}{\mathrm{V}_{\mathrm{T}}} \times \frac{\mathrm{V}_{\mathrm{RX}}}{\mathrm{V}_{\mathrm{RI}}} \text { (3) }
$$

In formula (3), $\mathrm{V}_{\mathrm{RI}}$ is the total volume within the reference isodose curve. The $\mathrm{CI}$ is between 0 and 1 , and higher $\mathrm{CI}$ values indicate better dose conformity. For the OARs, the average dose $D_{\text {mean }}$ and the near-maximum dose $\mathrm{D}_{2} \%$ to the PRV of brainstem and spinal cord were calculated, and the average dose $\mathrm{D}_{\text {mean }}$ for other OARs was calculated.

Within the same patient, the perturbation effect of the immobilization device on the entire treatment plan was calculated in the TPS by plan subtraction. The dose difference at a particular location $\mathrm{P}(\mathrm{x}, \mathrm{y}, \mathrm{z})$ in the patient was calculated as the dose difference between Plan+ and Plan- at this position:

$$
\text { Diff }(G y)=D(x, y, z)_{\text {Plan+ }}-D(x, y, z)_{\text {Plan- }}(4)
$$

For statistical analysis, the dose parameters of DVH for Plan- and Plan+ were compared using a t-test in SPSS 19.0 software (IBM Co., Armonk, New York, US); A difference with a two-side $p$ value of less than 0.05 indicated a statistically significant difference.

\section{Dose measurements}

An Alderson Radiation Therapy (ART) anthropomorphic phantom (RSD Inc., USA) and gafchromic EBT3 film (ASHLAND Inc., USA) were used to verify the dose distribution of Plan- and Plan+. The ART phantom is transected-horizontally into $2.5 \mathrm{~cm}$ thick slices and mimicks the head and neck anatomy of actual patients (Figure 1B). The EBT3 films used in this study were taken from the same batch (NO. 11091602) and the calibration dose range was 0-4 Gy. EBT3 film sheets were cut into pieces of required size (about $7.8 \mathrm{~cm} \times 14.5 \mathrm{~cm}$ ) for dose measurement and were sandwiched in the transverse slices of the phantom. To simulate the actual patient simulation procedure, the phantom was immobilized with the head and neck immobilization equipment. The irradiated films were scanned and imported into an in-house analysis software (QAchart) which used a verified triple-channal dose analysis method [22]. At commonly used gamma evaluation criteria (3\% dose difference (DD) and $3 \mathrm{~mm}$ distance to agreement (DTA)), the global gamma analysis was performed for evaluate the dose accuracy of patient Plan+. One particular point dose on the posterior neck skin of the phantom was measured from the irradiated EBT3 film and compared with the TPS calculations from Plan+ and Plan-.

\section{Results}

\section{Doses in the targets}

As shown in the DVH results from one typical patient (Fig. 1), when the nasopharyngeal carcinoma immobilization device was taken into account for treatment planning and dose calculation, the curve of each ROI in the Plan+ shifted to the left, and the PTV coverage rate for the prescription dose and mean dose (D $\left.D_{\text {mean }}\right)$ were lower than Plan-. In Table 1 , the DVH statistical analysis of 10 patients showed that the CRs of PTVnx, PTVnd, PTV1 and PTV2 were reduced by $2.4 \%, 9.9 \%, 1.5 \%$ and $3.6 \%$, respectively, and the reduction effect was highest for lymph node PTVnd. The $D_{\text {mean }}$ values of the four PTVs were decreased by $0.9 \%, 1.9 \%, 1.1 \%$ and $1.5 \%$, respectively, and these differences were statistically significant. The head and 
neck immobilization device had little effect on the dose HI of PTVnx and PTVnd but had strong effects on the CIs of each target; the differences in the four target areas were $11.1 \%,-1.9 \%, 6.0 \%$ and $2.9 \%$.

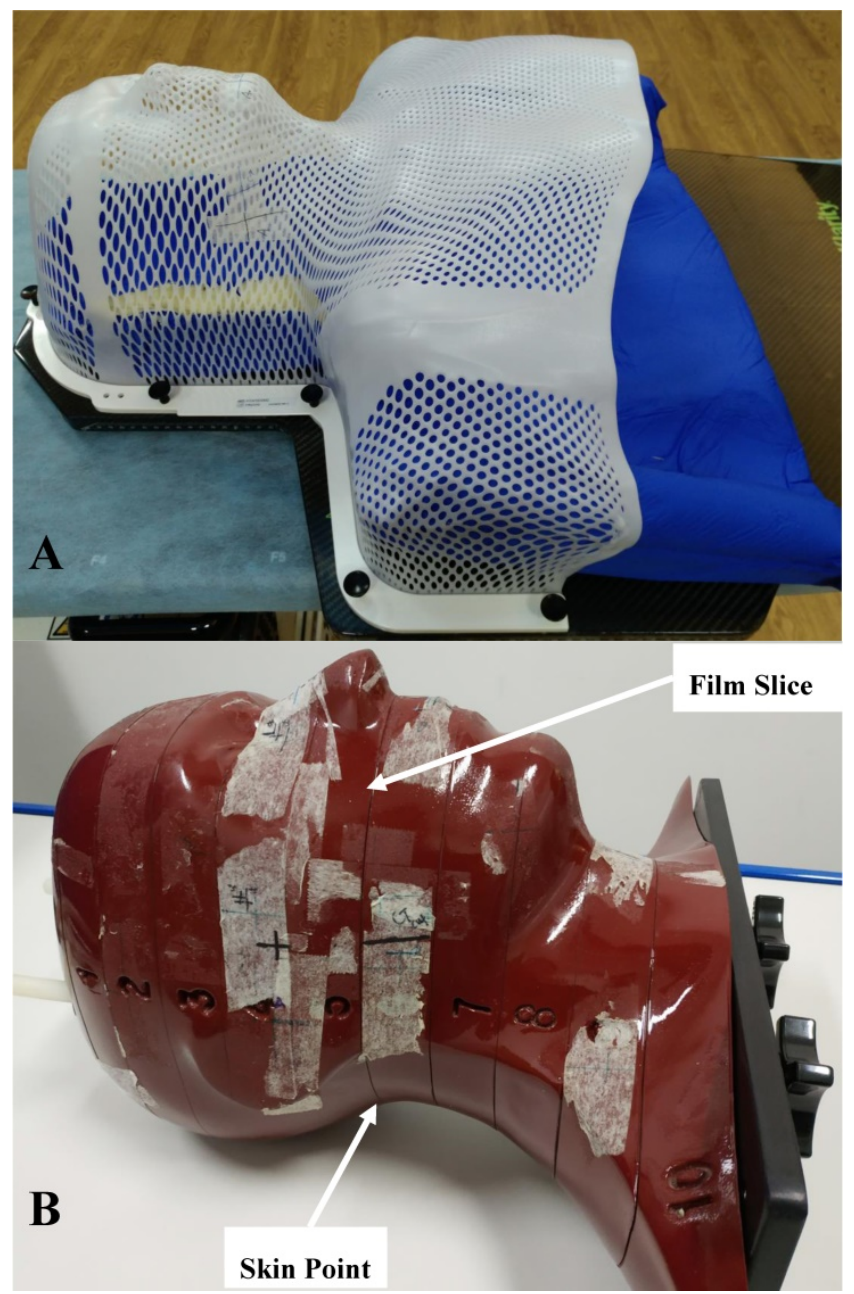

Figure 1. A: The head and neck immobilization device used for nasopharyngeal carcinoma radiation treatment. The white piece is the head, neck and shoulder fixation mask of thermoplastic. The blue piece is the head and neck rest of vacuumed styrofoam bag, and the black piece is the carbon fiber floor of the whole device; B: The anthropomorphic phantom used for the film dosimetry. The phantom is horizontally transected into $2.5 \mathrm{~cm}$ thick slices. Each slice has holes which are plugged with bone and soft tissue equivalent materials to mimick the head and neck anatomy of actual patients.

\section{OARs dose}

As shown in Table 2, the near-maximum dose was not significantly different in the spinal cord and brain stem. However, to other OARs, the $D_{\text {mean }}$ values were reduced due to the dose attenuation effect of the head and neck immobilization device. The maximum reduction in $\mathrm{D}_{\text {mean }}$ was $1.7 \%$, and the dose differences were statistically significant. Fig. 1 showing similar results, the brain stem and spinal cord DVH curves of the two plans did not differ significantly while other ROI curves did.
Table 1. Planning target volumes dosimetric parameters ( $\bar{x} \pm S$ ) of the two kinds of plans studied for 10 cases of nasopharyngeal carcinoma.

\begin{tabular}{llllll}
\hline Targets & Parameters & Plan + & Plan- & Diff $(\%)$ & $p$ value \\
\hline PTVnx & CR(\%) & $96.07 \pm 1.43$ & $98.32 \pm 0.89$ & $-2.35 \pm 1.21$ & $<0.01$ \\
& $D_{\text {mean }}(\mathrm{Gy})$ & $72.37 \pm 1.71$ & $73.04 \pm 1.55$ & $-0.93 \pm 0.32$ & $<0.01$ \\
& HI & $0.07 \pm 0.02$ & $0.07 \pm 0.02$ & 0 & NA \\
& CI & $0.76 \pm 0.09$ & $0.68 \pm 0.15$ & $11.14 \pm 13.55$ & 0.01 \\
PTVnd & CR(\%) & $89.90 \pm 7.10$ & $98.27 \pm 1.27$ & $-9.92 \pm 8.62$ & 0.01 \\
& D $($ mean $(G y)$ & $66.20 \pm 1.14$ & $67.47 \pm 1.09$ & $-1.92 \pm 0.38$ & $<0.01$ \\
& HI & $0.07 \pm 0.01$ & $0.07 \pm 0.01$ & $2.92 \pm 6.22$ & 0.17 \\
& CI & $0.15 \pm 0.08$ & $0.15 \pm 0.07$ & $-1.93 \pm 15.73$ & 1.00 \\
PTV1 & CR(\%) & $97.39 \pm 0.94$ & $98.85 \pm 0.54$ & $-1.51 \pm 0.69$ & $<0.01$ \\
& D mean(Gy) & $69.36 \pm 2.73$ & $70.09 \pm 2.58$ & $-1.07 \pm 0.40$ & $<0.01$ \\
& CI & $0.45 \pm 0.10$ & $0.43 \pm 0.09$ & $5.99 \pm 2.91$ & $<0.01$ \\
PTV2 & CR(\%) & $94.21 \pm 2.25$ & $97.56 \pm 0.90$ & $-3.60 \pm 2.60$ & $<0.01$ \\
& D $($ mean $(G y)$ & $60.39 \pm 1.10$ & $61.31 \pm 1.09$ & $-1.52 \pm 0.25$ & 0.01 \\
& CI & $0.79 \pm 0.02$ & $0.76 \pm 0.03$ & $2.89 \pm 3.54$ & 0.03 \\
\hline
\end{tabular}

Note: Diff $(\%)=(($ Plan +$)-($ Plan- $)) /($ Plan +$) \times 100$.

Abbreviations: Plan-, plan generated without immobilization device; Plan+, plan calculated from Plan- with immobilization device taken into accounted; PTV, planning target volume; $\mathrm{CR}$, coverage rate; $\mathrm{HI}$, homogeneity index; $\mathrm{CI}$, conformity index.

Table 2. Dose results of OARs between two kinds of plans in 10 cases of nasopharyngeal carcinoma $(\bar{x} \pm S)$.

\begin{tabular}{llllll}
\hline OARs & Parameters & Plan + & Plan- & Diff $(\%)$ & $p$ value \\
\hline PRV-BrainStem & $D_{\text {mean }}(\mathrm{Gy})$ & $32.32 \pm 3.17$ & $32.59 \pm 3.18$ & $-0.83 \pm 1.05$ & 0.03 \\
& $\mathrm{D}_{2 \%}(\mathrm{~Gy})$ & $55.83 \pm 3.42$ & $56.08 \pm 3.21$ & $-0.48 \pm 1.82$ & 0.47 \\
PRV-Spinal & $\mathrm{D}_{\text {mean }}(\mathrm{Gy})$ & $33.66 \pm 2.15$ & $33.94 \pm 2.18$ & $-0.86 \pm 0.31$ & $<0.01$ \\
& $\mathrm{D}_{2 \%}(\mathrm{~Gy})$ & $41.18 \pm 0.71$ & $41.53 \pm 0.86$ & $-0.83 \pm 1.31$ & 0.08 \\
Parotid & $\mathrm{D}_{\text {mean }}(\mathrm{Gy})$ & $38.67 \pm 2.23$ & $38.96 \pm 2.30$ & $-0.80 \pm 0.42$ & $<0.01$ \\
Tongue & $\mathrm{D}_{\text {mean }}(\mathrm{Gy})$ & $40.50 \pm 4.63$ & $41.19 \pm 4.66$ & $-1.70 \pm 0.54$ & $<0.01$ \\
Inner ear & $\mathrm{D}_{\text {mean }}(\mathrm{Gy})$ & $47.18 \pm 3.73$ & $47.59 \pm 3.86$ & $-0.84 \pm 0.83$ & 0.01 \\
Middle ear & $\mathrm{D}_{\text {mean }}(\mathrm{Gy})$ & $39.12 \pm 3.51$ & $39.60 \pm 3.64$ & $-1.22 \pm 0.68$ & $<0.01$ \\
Larynx & $\mathrm{D}_{\text {mean }}(\mathrm{Gy})$ & $45.55 \pm 3.38$ & $45.95 \pm 3.42$ & $-0.89 \pm 0.45$ & $<0.01$
\end{tabular}

\section{Note: $\operatorname{Diff}(\%)=(($ Plan +$)-($ Plan- $)) /($ Plan +$) \times 100$.}

Abbreviations: OAR, organ at risk; Plan-, plan generated without immobilization device; Plan+, plan calculated from Plan- with immobilization device taken into accounted; $\mathrm{D}_{\text {mean, }}$ mean dose. $\mathrm{D}_{2 \%}$, near-maximum dose.

\section{Dose difference distribution map (Plan+ - Plan-)}

In the TPS, the results from two plans of the same patient can be directly subtracted to calculate the dose difference distribution map for the patient. As shown in Figure 3, the blue to red gradient represented different absolute dose difference values ranging from -6 Gy to $30 \mathrm{~Gy}$. The dose distribution was dramatically altered due to the attenuation and bolus effect of the immobilization devices. In particular, as shown in B) and C), the neck dose near the head and neck immobilization device was significantly reduced, mainly due to the attenuation when the incident beam ray passes through the immobilization device. Changes in radiation scattering and build-up effect occurred due to the presence of low-density foam in the vacuum pad between the patient's neck and the floor plate. As a result, the dorsal neck skin dose was increased by approximately 10 Gy (Figure 3A, 3B). 


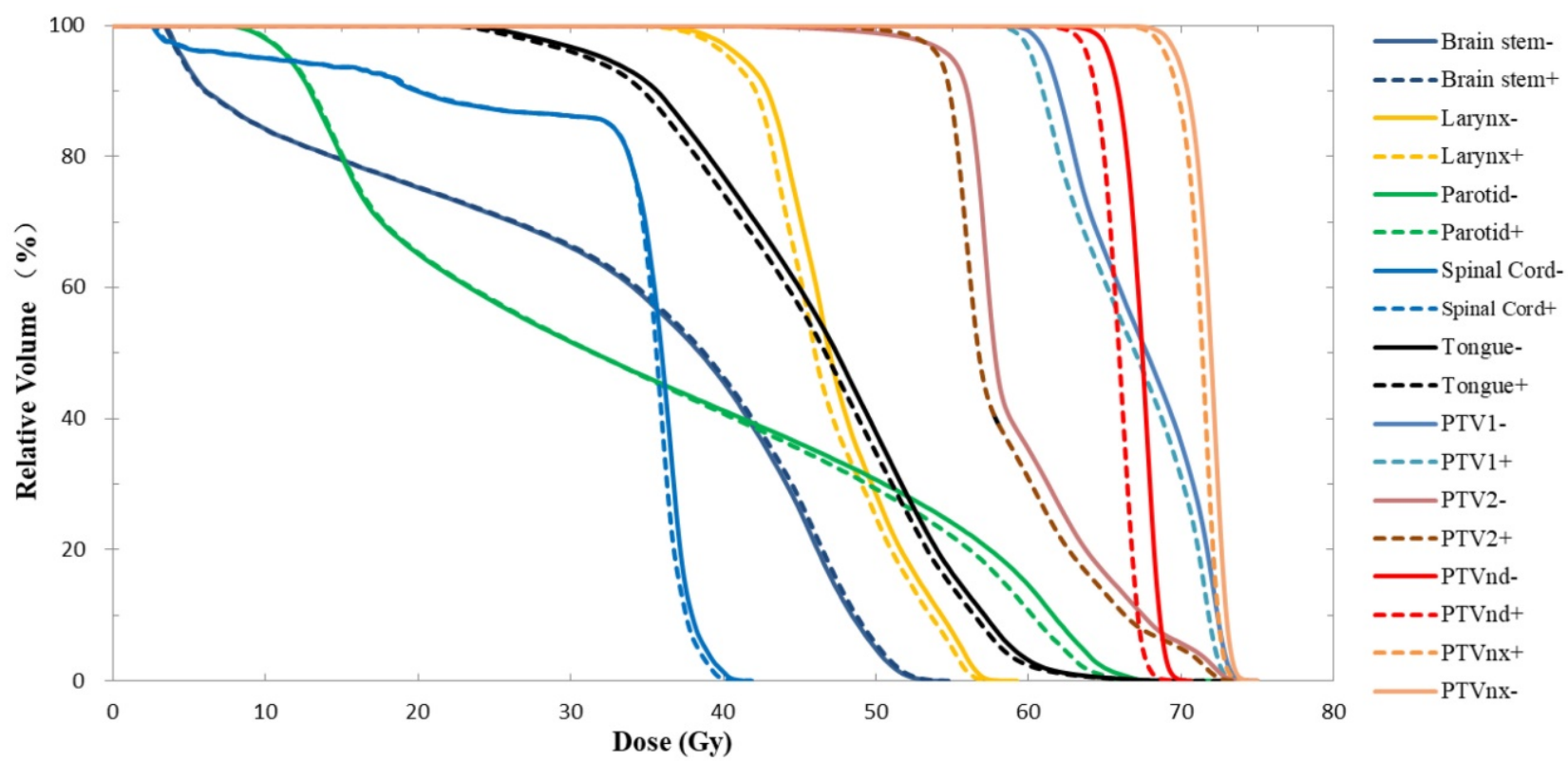

Figure 2. DVH results of Plan- and Plan+ for one typical patient with nasopharyngeal carcinoma. The solid lines represent the results of Plan- (calculated without immobilization device), and the dotted lines represent the results of Plan+ (calculated with the whole head and neck immobilization device included in the external body contour).

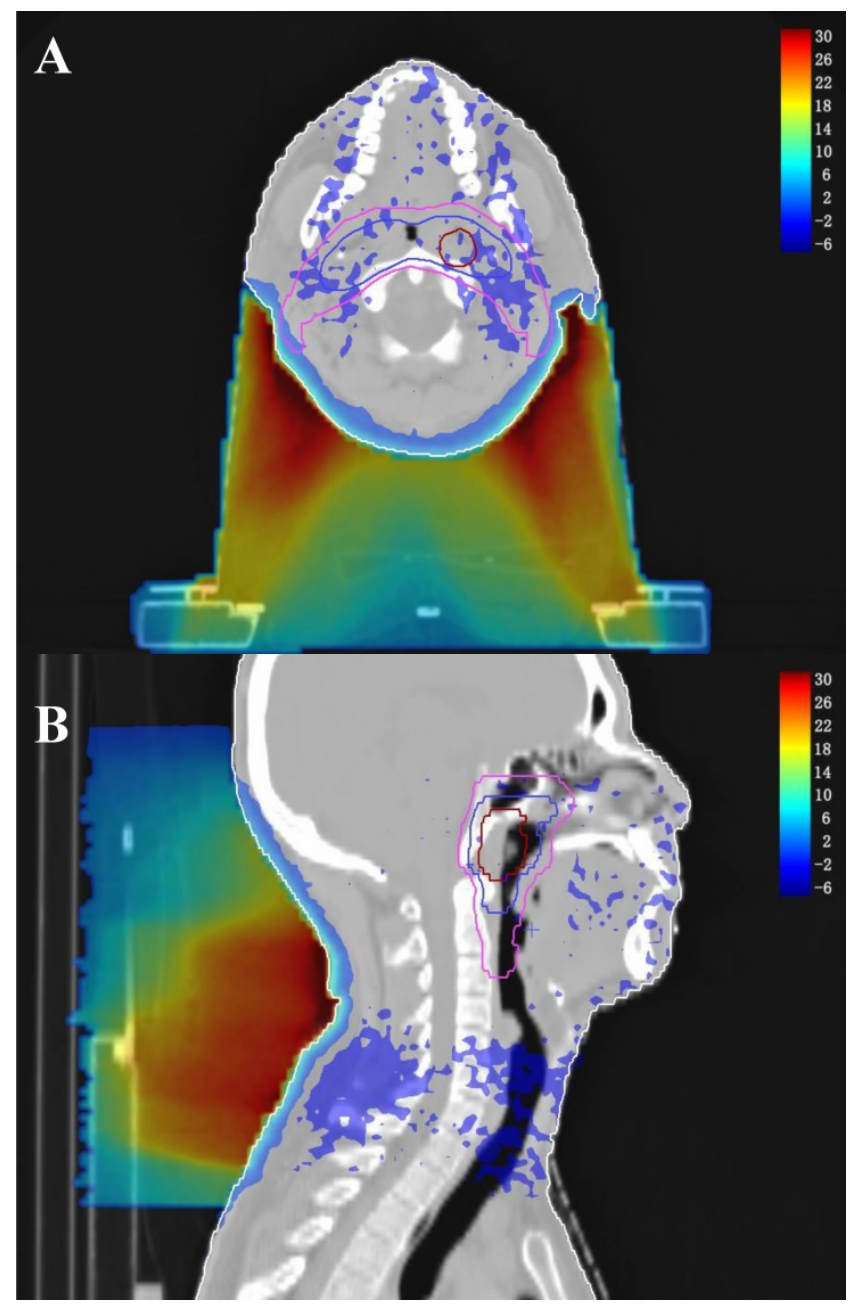

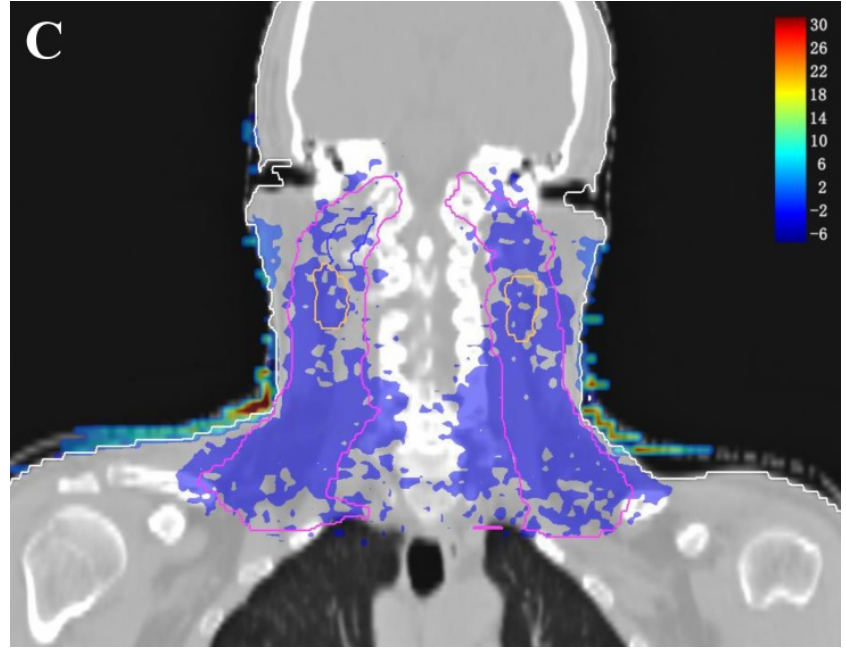

Figure 3. Three dimensional dose difference distribution map for a typical NPC case. Dose difference was calculated through subtracting Plan- from Plan+. (A: Cross-sectional plane, B: Sagittal plane, and C: Coronal plane).

\section{Dose measurement verification}

For Plan+ of ten NPC patients, the global percentage gamma passing rates were calculated at the setting dose difference $(3 \%)$ and $3 \mathrm{~mm}$ distance to agreement (DTA). The mean global gamma passing rate was $92.1 \pm 2.1 \%$. As Figure 4 showed, for a typical point on the patient posterior skin, the EBT3 measurement results $(23.0 \pm 3.7 \mathrm{~Gy})$ were more close to the Plan+ calculations $(23.3 \pm 3.4$ Gy) than Planresults $(15.3 \pm 2.7 \mathrm{~Gy})$. The Plan-, which calculated the dose distributions without including the immobilization device into external contour, underestimated the skin dose about $53 \%$. 


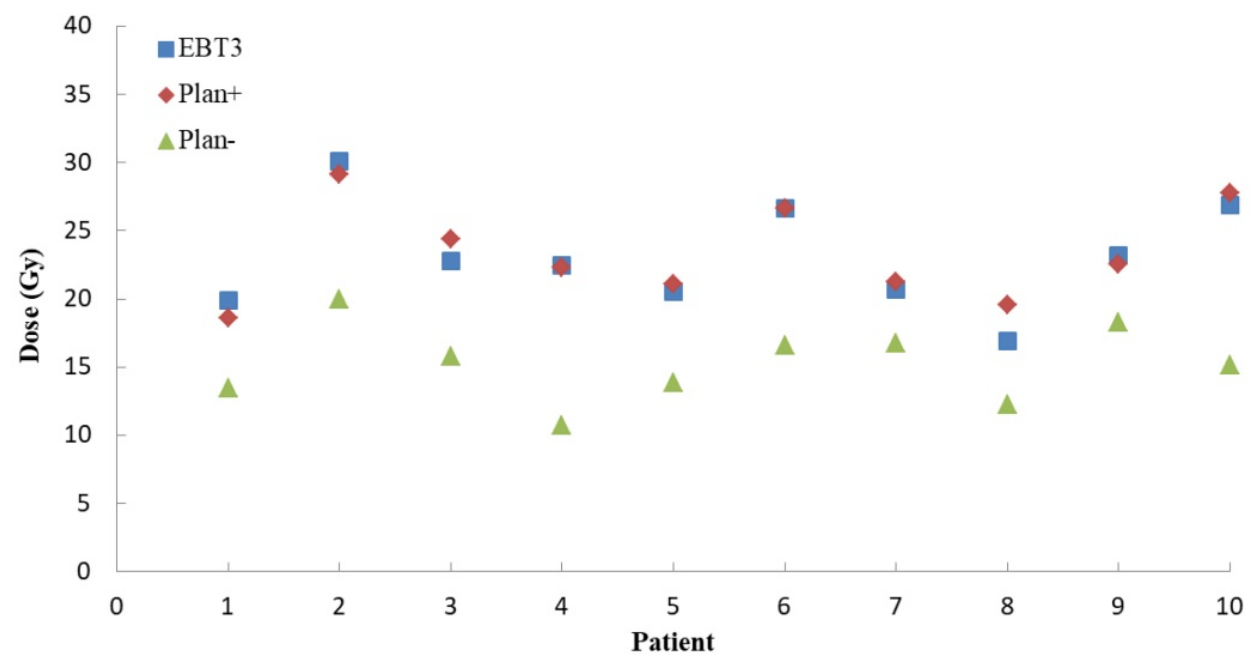

Figure 4. The measurements and TPS calculations point doses on posterior skin for ten NPC patients. The blue squares represent the doses EBT3 film measurement. The red diamonds are the Plan+ (dose calculating with immobilization devices) doses and the green triangles represent the Plan- (dose calculating without immobilization devices) doses.

\section{Discussion}

Patient immobilization devices and other treatment accessories used in radiotherapy are not completely radio-translucent and can affect the radiation dose during the delivery of treatment [16]. The measurement of the single fixed beam indicated that the attenuation effect and the bolus effect could change significantly when the radiation beam passed through the treatment bed, polymethyl methacrylate (PMMA) and other objects in the path of the beam. Pulliam KB et al. found the treatment couch caused average prescription dose losses (relative to plans that ignored the couch) to the prostate of $4.2 \%$ and $2.0 \%$ for IMRT with the rails out and in, respectively, and the percentage of the target covered by the prescribed dose dropped to $35 \%$ and $84 \%$ for IMRT (rails out and in, respectively) [7]. Tsang et al. found the treatment through a vacuum compressed immobilization device (vacuum bag) increased the dose delivered to the skin by interactions of the $X$-rays within the vacuum bag material. At a $10 \mathrm{~cm} \times 10 \mathrm{~cm}$ field the dermal skin layer ( $1 \mathrm{~mm}$ depth) doses increased from $44 \%$ (no bag) to $60 \%$ for a bag thickness of $2.5 \mathrm{~cm}$ at $6 \mathrm{MV}$ X-rays [23]. Puysseleyr et al. measured the dosimetric impact of a prone breast immobilization device and found that beam attenuation amounted to 7.6\% (6 MV X-ray) for beam obliquely intersecting the couch top-baseplate combination and $12.3 \%$ beam attenuation for the radiation beam passed through the support wedge. Inclusion of the immobilization device components in the treatment planning CT allowed TPS to accurately model the most important attenuation effect [13].

The establishment of a virtual model (including geometry and density information) for the treatment couch top within the TPS is a common method used to calculate the attenuation effect of the treatment couch on treatment planning $[6,11,12]$. In this study, we contoured the head and neck immobilization devices into the external body contour to calculate the immobilization device impact on the dose distribution of NPC IMRT. The density information of the immobilization devices showed the TPS calculation algorithm was able to compute dose for the situation with the immobilization devices enclosed. Due to the attenuation of the immobilization devices, CRs and $\mathrm{D}_{\text {mean }}$ in Plan+ were reduced compared to those in Plan- (the prescription dose coverage rates of the four targets were decreased by $1.5-9.9 \%$, and the average doses were decreased by $0.9-1.9 \%$ ). The attenuation effect was greater on the dose coverage rates of PTVnd and PTV2 $(-9.9 \%$ and $-3.6 \%$, respectively), mainly because the two PTVs were spatially closer to the immobilization device. Table 1 showed that the Plan+ prescription dose coverage volumes for PTVnd and PTV2 were less than 95\%, and according to the protocols of nasopharyngeal carcinoma IMRT planning in our center, these plans are not suitable for clinical treatment. Among the doses in OARs, except the near-maximum doses for the brain stem and spinal cord PRV had no significant difference, the average doses of all other OARs were statistically significantly reduced (with a maximum difference of $1.7 \%$ ). Figure 2 showed a similar result; the DVH curves of the brain stem and spinal cord did not show significant differences between the two plans, but the curves for each PTV exhibited a certain degree of separation. The dose difference maps in Figure 3 also showed that due to the presence of the head and neck immobilization device, the dose distribution in the patient was reduced compared to that when the immobilization device was not considered. In particular, the dose in the portion of the lower neck 
that is close to the head and neck immobilization device was significantly reduced (most of which is in the PTVnd and PTV2 areas), mainly due to the attenuation effect of incident radiation passing through the immobilization device.

The importance of skin dose is often overlooked when megavoltage photon beams are used, but clinically relevant skin toxicity due to the beam passage through the treatment couch top has been reported [24,25]. Archambeau et al. found that in conventional fractionated radiotherapy (2 Gy per fraction), a skin dose greater than 25 Gy could produce clinically relevant skin reactions, and greater than 45 Gy could cause dry desquamation [26]. Hoppe et al. reported that in stereotactic body radiotherapy (SBRT) for early non-small cell lung cancer, due to the omission of the increased skin dose caused by the treatment couch top and immobilization device, 38\% of patients experienced acute skin radiotoxicity, 2 patients experienced grade 3 skin toxicity, and 1 patient experienced grade 4 skin toxicity [24]. So, the skin dose changes should be taken into account when the treatment beams passing through the couch top and immobilization devices. Our results in Figure 3 show that the presence of low-density polystyrene foam between the patient's neck and the floor plate resulted in a bolus effect of the radiation beam and a significant increase in the dorsal neck skin dose of approximately $8 \mathrm{~Gy}$. It should be noted that increasing the MU to correct for immobilization device attenuation may further increase the absolute skin dose. EBT3 film measurement results demonstrated that the Plan+ dose distribution was more close to the measurements. In Fig 4, for the patient posterior skin dose, the Plan+ calculations $(23.3 \pm 3.4 \mathrm{~Gy})$ were more close to the measurement $(23.0 \pm 3.7$ Gy) than Plan- calculations $(15.3 \pm 2.7$ Gy). The Plan- dramatically underestimated the posterior neck skin dose about 53\%. Therefore, it is recommended that the head and neck immobilization device should be contoured within the human body structure in nasopharyngeal carcinoma treatment planning, and the increase in skin dose caused by the immobilization device should be fully considered in order to yield a better dose calculation accuracy in the TPS.

\section{Conclusion}

In this study, we calculated and evaluated the dosimetric effects of the head and neck immobilization devices on the dose distributions of multi-field IMRT for NPC. The reduction in prescription dose coverage rate and mean dose to the target volumes were considerable, especially to PTVnd and PTV2. The increase in the skin dose could be up to $53 \%$ on average. The immobilization device should be included within external body contour to account for the dose attenuation and skin dose increment in the TPS calculation.

\section{Abbreviations}

IMRT: Intensity-modulated radiation therapy; NPC: nasopharyngeal carcinoma; TPS: Treatment planning system; GTV: Gross target volume; CTV: Clinical target volume; PTV: Planning target volume; AAPM: American Association of Physicists in Medicine; OAR: Organ at risk; PRV: Planning organ-at-risk volume; DVH: Dose volume histogram; CR: Coverage rate; HI: Homogeneity index; CI: Conformity index; DD: Dose difference; DTA: Distance to agreement; PMMA: Polymethyl methacrylate; SBRT: Stereotactic body radiotherapy.

\section{Acknowledgements}

This work was jointly supported by the National Key R\&D Program of China (2017YFC0113200), Science and Technology program of Guangdong Province, China (2015B020214002) and Science and Technology program of Guangzhou, China (201508020105).

\section{Availability of data and materials}

The datasets are backed up on the Research Data Deposit (RDD, https://www.researchdata.org.cn, approval number: RDDA2017000231) and are available on reasonable request.

\section{Authors' contributions}

LC and YP conceived and designed this study and drafted the manuscript under the supervision of $\mathrm{XD}$. XD participated in the design and coordination of the study, and helped to draft the manuscript. SG and HS selected the patient collective and contoured the reigns of interest. SG and DZ conducted IMRT plans. HS, WS and JW collected the data and performed the analysis. All authors read and approved the final manuscript.

\section{Competing Interests}

The authors have declared that no competing interest exists.

\section{References}

1. Contesini $M$, Guberti $M$, Saccani $R$, et al. Setup errors in patients with head-neck cancer (HNC), treated using the Intensity Modulated Radiation Therapy (IMRT) technique: how it influences the customised immobilisation systems, patient's pain and anxiety. Radiat Oncol. 2017; 12: 72.

2. Xu SK, Yao WY, Hu J, et al. The research of accuracy immobilized using individualized polyurethane scaling agent compared to positioning foam with standard plastics pillow in the radiotherapy of nasopharyngeal carcinoma. Chinese Journal of Radiation Oncology. 2015; 24: 196-199. 
3. Tang JM, Ma XM, Hou Y L,et al. Analysis of simultaneous modulated accelerated radiotherapy (SMART) for nasopharyngeal carcinomas. J Radiat Res. 2014; 55: 794-802.

4. Pan $\mathrm{XB}$, Huang $\mathrm{ST}$, Chen $\mathrm{KH}$, et al. Intensity-modulated radiotherapy provides better quality of life than two-dimensional conventional radiotherapy for patients with stage II nasopharyngeal carcinoma. Oncotarget. 2017; 8: 46211-18.

5. Wu S, Xie C, Jin $\mathrm{X}$, et al. Simultaneous modulated accelerated radiation therapy in the treatment of nasopharyngeal cancer: A local center's experience. Int J Radiat Oncol Biol Phys. 2006; 66 (Suppl 4): S40-S46.

6. Vanetti E, Nicolini G, Clivio A, et al. The impact of treatment couch modelling on RapidArc. Phys Med Biol. 2009; 54: N157-66.

7. Pulliam KB, Howell RM, Followill D, et al. The clinical impact of the couch top and rails on IMRT and arc therapy. Phys Med Biol. 2011; 56: 7435-47.

8. Olch AJ, Lavey RS. Reproducibility and treatment planning advantages of a carbon fiber relocatable head fixation system. Radiother Oncol. 2002; 65: 165-8.

9. McCormack S, Diffey J, Morgan A. The effect of gantry angle on megavoltage photon beam attenuation by a carbon fiber couch insert. Med Phys. 2005; 32: 483-7.

10. Njeh CF, Raines TW, Saunders MW. Determination of the photon beam attenuation by the Brainlab imaging couch: angular and field size dependence. J Appl Clin Med Phys. 2009; 10: 2979.

11. Mihaylov IB, Corry P, Yan Y, et al. Modeling of carbon fiber couch attenuation properties with a commercial treatment planning system. Med Phys. 2008; 35: 4982-88.

12. Gerig LH, Niedbala M, Nyiri BJ. Dose perturbations by two carbon fiber treatment couches and the ability of a commercial treatment planning system to predict these effects. Med Phys. 2010; 37: 322-8.

13. De Puysseleyr A, De Neve W, De Wagter C. A patient immobilization device for prone breast radiotherapy: Dosimetric effects and inclusion in the treatment planning system. Phys Med. 2016; 32: 758-66.

14. Munjal RK, Negi PS, Babu AG, et al. Impact of $6 \mathrm{MV}$ photon beam attenuation by carbon fiber couch and immobilization devices in IMRT planning and dose delivery. J Med Phys. 2006; 31: 67-71.

15. Lee $\mathrm{KW}, \mathrm{Wu} \mathrm{JK}$, Jeng $\mathrm{SC}$, et al. Skin dose impact from vacuum immobilization device and carbon fiber couch in intensity modulated radiation therapy for prostate cancer. Med Dosim. 2009; 34: 228-232.

16. Olch AJ, Gerig L, Li H, et al. Dosimetric effects caused by couch tops and immobilization devices: report of AAPM Task Group 176. Med Phys. 2014; 41: 061501.

17. Landberg T, Chavaudra J, Dobbs J, et al. Prescribing, Recording and Reporting Photon Beam Therapy: ICRU Report 50. Journal of the International Commission on Radiation Units and Measurements. 1993; os26: NP. Https://doi.org/10.1093/jicru/os26.1.Report50

18. Landberg T, Chavaudra J, Dobbs J, et al. Prescribing, Recording and Reporting Photon Beam Therapy (Supplement to ICRU Report 50): ICRU Report 62. Journal of the International Commission on Radiation Units and Measurements. 1999; os32: NP. Https://doi.org/10.1093/jicru/ os32.1.Report62.

19. Oliver M, Chen J, Wong E, et al. A treatment planning study comparing whole breast radiation therapy against conformal, IMRT and tomotherapy for accelerated partial breast irradiation. Radiother Oncol. 2007; 82: 317-23.

20. Nakamura JL, Verhey LJ, Smith V,et al. Dose conformity of gamma knife radiosurgery and risk factors for complications. Int J Radiat Oncol Biol Phys. 2001; 51: 1313-19.

21. [No authors listed]. ICRU Report 83: Prescribing, Recording, and Reporting Photon-Beam Intensity-Modulated Radiation Therapy (IMRT). Oxford, UK: Oxford University Press; 2010.

22. $\mathrm{Li} \mathrm{Y}$, Chen $\mathrm{L}$, Zhu J, et al. The combination of the error correction methods of GAFCHROMIC EBT3 film. PLoS One. 2017; 12: e0181958.

23. Cheung T, Butson MJ, Yu Peter KN. Evaluation of build-up dose from $6 \mathrm{MV}$ X-rays under pelvic and abdominal patient immobilization devices. Radiat Meas. 2002; 35: 235-238.

24. Hoppe BS, Laser B, Kowalski AV, et al. Acute skin toxicity following stereotactic body radiation therapy for stage I non-small-cell lung cancer: who's at risk? Int J Radiat Oncol Biol Phys. 2008; 72(5):1283-6.

25. Lee N, Chuang C, Quivey JM, et al. Skin toxicity due to intensity-modulated radiotherapy for head-and-neck carcinoma. Int J Radiat Oncol Biol Phys. 2002; 53(3):630-7.

26. Archambeau JO, Pezner R, Wasserman T. Pathophysiology of irradiated skin and breast. Int J Radiat Oncol Biol Phys. 1995; 31(5): 1171-85. 